\title{
Diagnosis and treatment of food allergies
}

\section{By J. B. Anderson* and M. H. Lessof, Department of Medicine, Guy's Hospital Medical School, London $S E_{\mathrm{I}}{ }_{9} R T$}

\section{Clinical classification of food allergy}

Food allergy has become a contentious subject. True food allergy is dependent on evidence of intolerance to a specific food-in other words recurrence of symptoms on two or more occasions when that food is taken (preferably blind and in the presence of the doctor). There should also be direct or indirect evidence of an immunological component to that reaction.

Patients may have 'simple' intolerance to foods rather than true allergy for various reasons. For example, the patient who develops tachycardia while drinking ten strong cups of coffee daily has a pharmacological cause of food intolerance. The patient with food-induced migraine may in some instances have low phenol sulphotransferase levels. Thus an enzyme defect may be misinterpreted as an allergy. If we are to study well-defined groups we should not talk of food allergy in these cases any more than we should for a patient with gallstones who cannot eat eggs. Furthermore, food allergy is frequently applied to people who for one reason or another have a behavioural aversion to foods without any objective evidence that that food upsets them at all. The correct diagnosis in such cases may range from anorexia nervosa or food fads to hyperventilation syndrome. The following represents a clinical classification of food allergy based on the experience of the Guy's Hospital Allergy Clinic.

Alimentary tract reactions. These can be divided into:

(I) Oropharyngeal reactions.

(2) Lower gastrointestinal reactions, e.g. nausea, vomiting and diarrhoea.

The former is perhaps the most commonly experienced food allergy. It frequently, but not necessarily occurs in the atopic individual and is manifest by the development of urticarial swelling and itching in and around the mouth minutes after eating specific foods. There is sometimes a feeling of swelling in the throat but very rarely is this severe enough to obstruct normal breathing. Although some such reactions are non-immunological reactions to chemical constituents of food (e.g. histamine) the diagnosis of allergy can be made if the patient has positive skin prick tests to the food concerned. The foods most commonly involved are nuts, citrus fruits, uncooked vegetables, eggs and cheeses.

Lower gastrointestinal reactions are less common. Characteristically the patient describes the development of nausea and vomiting with or without diarrhoea within $30 \mathrm{~min}$ of ingesting a specific food. This may be associated with more

- Present address: Department of Thoracic Medicine, New Cross Hospital, Avonley Road, London SE I4. $_{4}$ 
generalized symptoms, for example flushing and itching of the skin, headache and palpitations. The symptoms resolve spontaneously over $1-2 \mathrm{~h}$. The foods commonly implicated are seafoods, eggs and nuts.

Reactions in distant organs. The next major clinical grouping embraces all reactions occurring in distant organs after the ingestion of a specific food. This may involve a single organ, for example the development of generalized urticaria after eating a particular food. However, in patients who have generalized severe allergic disease with perhaps a long history of asthma, hay fever or perennial rhinitis, the gut may represent one of the portals by which allergens gain entry to the body. Such patients may describe aggravation of their asthma by a particular food developing within $30 \mathrm{~min}$ of its ingestion. The reaction may persist for some hours unless the target organ itself is treated appropriately. We have witnessed asthma secondary to food challenge and the reaction can be every bit as severe as that secondary to inhaled allergen challenge.

The diseases which are thought to be aggravated by food allergies are asthma, rhinitis, urticaria, eczema and migraine: the foods commonly implicated are eggs, cheese, milk, nuts and seafood.

Popular-press food allergy syndrome. The third major clinical group is that which I shall describe as the 'popular-press food allergy syndrome'. It has been claimed by some authors (Mackarness, 1976) that certain foods (particularly those which we like) are the underlying cause of many disparate symptoms, for example, lethargy, depression and 'bloating': there is no good evidence that they are. Nonetheless, this group, although not experiencing true food allergy, represents perhaps $50 \%$ of those patients presenting with food allergy to the clinic and their management may be difficult.

\section{Investigations}

Investigating patients with food allergy requires a thorough history of the patient's symptoms, the foods which they feel aggravate their disease, their previous medical and psychiatric history (with particular regard to any previous allergic history). Examination of the patient is necessary to exclude underlying disease which might otherwise explain their symptoms (e.g. thyrotoxicosis causing anxiety and diarrhoea).

Routine investigations include a full blood count and differential white cell count, total serum IgE analyses, and skin sensitivity tests (including those specific to the foods under consideration). If the target organ is distant from the gastrointestinal tract it may be necessary to investigate this separately, e.g. peak expiratory flow rates (PEFR) for food-induced asthma. In those patients for whom the offending food is not apparent, a food exclusion diet may be used as a diagnostic tool. Food challenge experiments in the presence of the investigating physician remain the most useful investigative technique.

Obtaining objective evidence of food allergy may be difficult. Nonetheless, it is a necessary part of research investigation and a desirable ideal of clinical 
investigation. The investigations themselves are tailored according to the category of food allergy.

Alimentary tract reactions. Immediate oropharyngeal reactions require the least investigation as the patient readily identifies the offending foods: simple challenges with those foods corroborate the history and allow some evaluation of the extent and severity of the reaction. However, those reactions in the lower gastrointestinal tract are more difficult to evaluate. Simple challenge by eating the suspect food is less satisfactory than blind challenge via a nasogastric tube because the patient may anticipate their own response to that food. If that response is merely symptomatic, e.g. nausea or abdominal pain, it is difficult to evaluate. However, if the food is given blind through a nasogastric tube after a succession of nonallergenic challenges (e.g. with water, saline, etc.), a symptomatic response is more credible. We have utilized this technique in our department, particularly in the investigation of patients with food-induced abdominal symptoms and foodinduced asthma. It affords a reproducible technique whereby patients and therapies may be evaluated. We have used plasma prostaglandin assays as a marker of food-allergic reactions (Buisseret et al. 1978) and used blind nasogastric challenge to assess the efficacy of prostaglandin inhibitors.

We challenged ten patients with gastrointestinal symptoms in this way with the specific food to which they were said to be sensitive. Of ten patients there were only positive clinical reactions in three, i.e. the development of abdominal symptoms following that food challenge. Of six asthmatics challenged in this way four had significant reactions, i.e. a fall in PEFR greater than $15 \%$ of the baseline value.

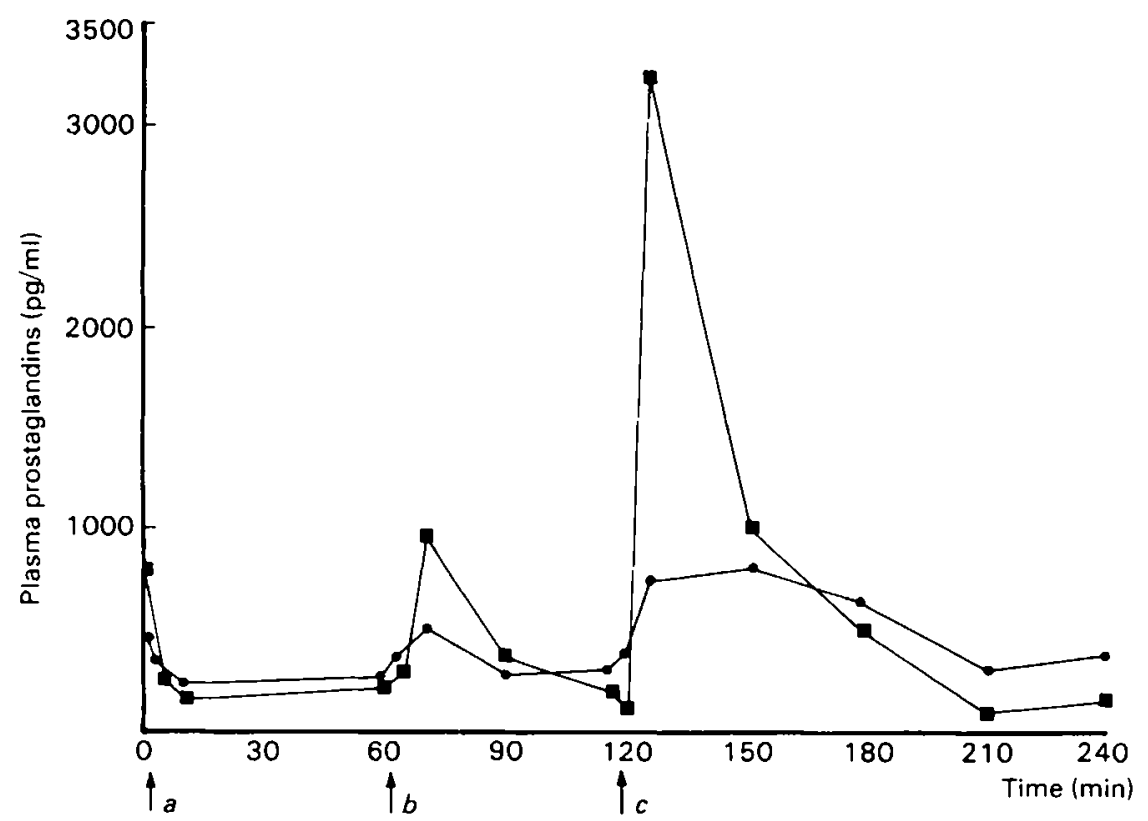

Fig. I. Plasma prostaglandin $\left(\square, \mathrm{PGF}_{2} \alpha ; 0, \mathrm{PGE}_{2}\right)$ response to direct gastric challenge with (a) $15^{\circ} \mathrm{ml}$ water, (b) $15^{\circ} \mathrm{ml}$ buffer solution and $(c) 150 \mathrm{ml}$ milk. The patient was said to be milk allergic. 


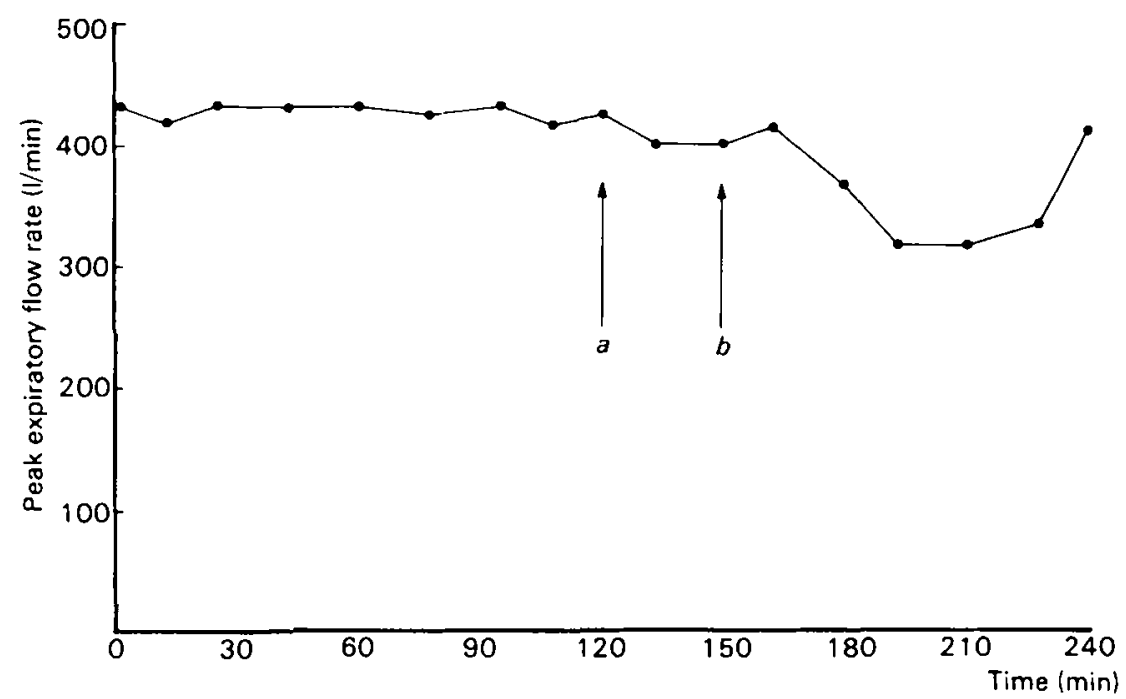

Fig. 2. The peak expiratory flow rate in response to crushed brazil nuts, $(a) 0.25 \mathrm{~g}$ and $(b) 0.5 \mathrm{~g}$, infused directly into the stomach via a nasogastric tube. The subject was a known asthmatic.

Figure 1 shows the plasma concentrations of prostaglandins $\mathrm{PGF}_{2} \alpha$ and $\mathrm{PGE}_{2}$ in response to a milk challenge in a man who reported abdominal pain and diarrhoea after drinking milk. Challenge was carried out on two separate occasions, initially without and subsequently with aspirin $\mathrm{I} h$ before the milk challenge. The prostaglandin response and concomitant symptoms were abolished by administering aspirin.

All patients who have diarrhoea as a suspected consequence of food sensitivity should be vigorously investigated to exclude other organic disease, as food allergy is a rare cause of diarrhoea.

Reactions in distant organs. The same basic principles apply to the investigation of food-induced rhinitis, asthma, etc. as to food-induced abdominal symptoms. The offending foods are first identified with or without the aid of a food exclusion diet. Blind nasogastric challenge is then performed using a peak nasal inspiratory flow meter to assess the development of an acute rhinitis or an asthmatic reaction. Figure 2 demonstrates the PEFR response to brazil-nut challenge in a male asthmatic.

Popular-press food allergy syndrome. It is important to make a positive diagnosis of this group of patients, partly to avoid unnecessary and inappropriate treatment, but also because there may be other forms of treatment appropriate to the underlying condition. For example, psychiatric referral for those with anorexia nervosa or depression. Patients commonly complain of multiple symptoms in different systems related to the ingestion of many different foods. Careful assessment of the patient's personal history may reveal that the diagnosis of 'allergy' and the apparent reactions associated with it afford the patient 'secondary gain' within the dynamics of their family life. Nonetheless, many patients simply identify themselves with popular-press descriptions of food allergy, which embrace 
so many facets of psychological and physical disease that we could all find some feature applicable to ourselves. The majority of such patients are gratified to learn that whatever problems they have, they can at least enjoy their Sunday lunch.

The allocation of a patient to this clinical group is usually obvious after taking a detailed history but, if in doubt, an exclusion diet should be embarked on. If this appears to identify specific foods triggering the patient's symptoms, this should be established by challenge testing--preferably blind.

\section{The treatment of food allergy}

The treatment of food allergy depends on the clinical category concerned. Nonetheless, the basis of all treatment is avoidance of the offending foods.

Treatment of alimentary tract reactions. Reactions occurring in the oropharynx invariably respond rapidly to treatment with antihistamines. Terfenadine (Triludan ${ }^{\circledR}, 60 \mathrm{mg}$ ) is recommended as it has a negligible sedative effect and should be taken immediately any reaction develops. It can also inhibit the development of such reactions if taken prophylactically, thereby allowing patients a free diet. Reactions in the lower gastrointestinal tract are less easily managed. In some patients prophylactic use of antihistamines or salicylates may inhibit the development of vomiting or diarrhoea, but long-term ingestion of salicylates, particularly, is not recommended. There is some evidence that ingested sodium cromoglycate is useful in such patients (Wraith et al. 1979). Again, the mainstay of treatment is avoidance of those foods which trigger symptoms. The patient is advised to avoid such foods but to assess their own sensitivity, perhaps annually, by challenging themselves with small amounts of that food.

Treatment of reactions in distant organs. The management of patients with food-induced urticaria, eczema, rhinitis or asthma is based initially on food avoidance and thereafter on appropriate treatment of the organ or system concerned. It is no more sensible to manage asthma that is triggered by inhaled allergens (for example Dermatophagoides pteronyssinus, the house-dust mite), by allergen avoidance alone than it is to manage food-induced asthma by allergen avoidance alone. To take asthma as an example, the woman whose PEFR response to yeast-extract challenge is shown in Fig. 3, had profound reactions to various inhaled and ingested allergens. When challenged with yeast extract, her PEFR fell from over $400 \mathrm{l} / \mathrm{min}$, which it had been consistently for over $2 \mathrm{~h}$, to nearly 100 $1 / \mathrm{min}$ Io min after infusing $150 \mathrm{ml}$ of diluted yeast-extract through a nasogastric tube. Her asthma partly responded to food exclusion in that she no longer had acute, profound episodes of wheezing in relation to particular foods. She was concurrently on 'standard' therapy for her asthma, i.e. Salbutamol $200 \mu \mathrm{g}$ four times a day and Beclomethasone dipropionate roo $\mu \mathrm{g}$ four times a day, both given by inhaler. However, thrice daily measurements demonstrated that she still had significant diurnal variation in her PEFR, a sign that her asthma was inadequately controlled. On an increased dose of bronchodilators the diurnal variation in PEFR disappeared and she has now been symptom-free for over 9 months.

The management of any allergic condition is only partly achieved by the 


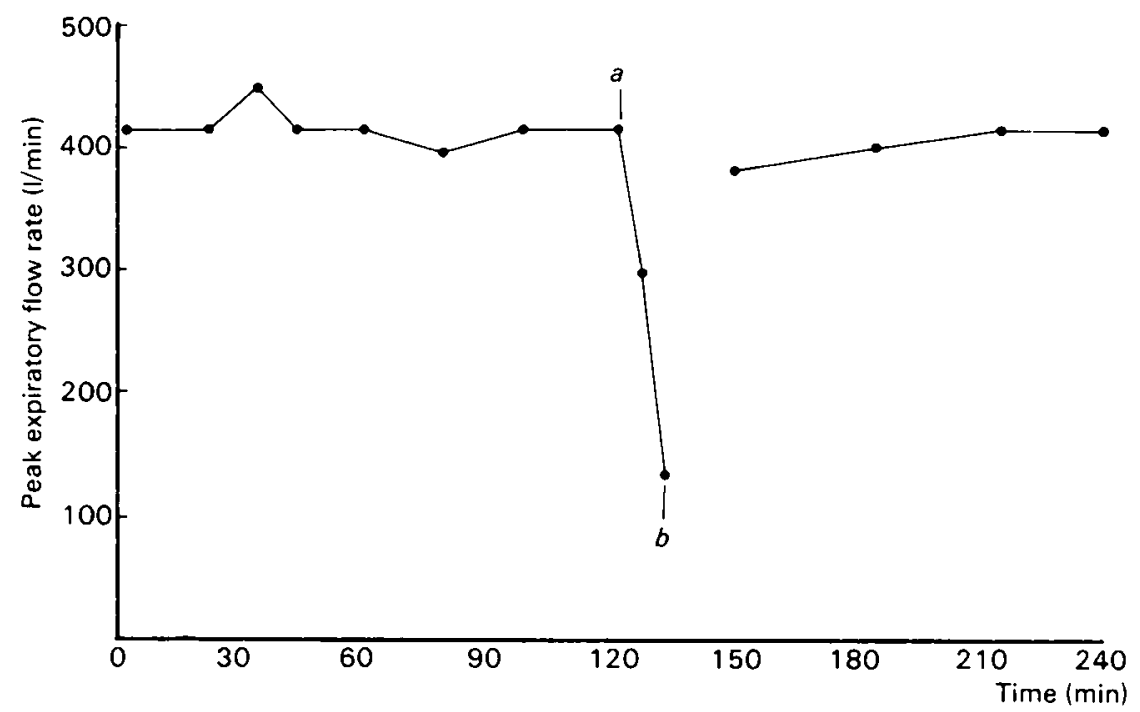

Fig. 3. The peak expiratory flow rate in response to $(a) 2 \mathrm{~g}$ yeast extract infused directly into the stomach via a nasogastric tube. The subject was a known asthmatic. (b) Treatment was $40 \%$ oxygen, $10 \mathrm{mg}$ Salbutamol and $150 \mathrm{mg}$ hydrocortisone.

identification and avoidance of specific allergens. It is completed by effective treatment of the target organ concerned.

Treatment of the popular-press food allergy syndrome. Although their symptoms may be real, the majority of patients in this group are grateful if, after listening to them sympathetically and reviewing the investigations performed, they are told that their symptoms are not related to their diet. A smaller number do have other medical conditions that explain their symptoms, for example, thyrotoxicosis. These should obviously be appropriately treated. If psychological disease is suspected (e.g. anorexia nervosa or depression) psychiatric referral is appropriate. However, there remains a small number of patients who appear determined that they have food allergy despite all evidence to the contrary.

To conclude, food allergy is relatively uncommon in the overall picture of allergy. Nevertheless, its diagnosis and treatment is increasingly part of the management of allergic disease.

\section{REFERENCES}

Buisseret, P. D., Youlten, L. J. F., Heinzelmann, D. I. \& Lessof, M. H. (1978). Lancet i, go6. Mackarness, R. (1976). Not All in The Mind. London: Pan Books.

Wraith, D. G., Young, G. V. W. \& Lee, T. H. (1979). Proceedings of the Mast-Cell International Symposium, Davos, pp. 443-6. 